\title{
Changes in gait pattern during multitask using smartphones
}

\author{
SoYeong Jeon, ChoRong Kim, SunHae Song and GyuChang Lee* \\ Department of Physical Therapy, Kyungnam University, Changwon-si, Gyeongsangnam-do, Republic of Korea
}

Received 15 January 2014

Accepted 2 June 2014

\begin{abstract}
.
BACKGROUND: With the development of science and technology, smartphones have been deeply involved in people's everyday lives, and many perform various tasks simultaneously on smartphones.

OBJECTIVE: To investigate gait pattern changes on performing multitask simultaneously when using smartphones.

METHODS: Three tasks were performed by 26 healthy adults. In the first, participants were directed to walk without using smartphones (single-task). In the second, they were required to walk while finding applications (dual-task). Lastly, in addition to performing the second task, they were asked to listen to questions and answer them on their smartphone (triple-task). Spatiotemporal variables of gait and degree of lateral deviation during walking were measured.

RESULTS: The results showed that there was a significant difference between the single-task and dual tasks, as well as between the single task and triple task in all variables $(p<0.05)$. In particular, gait velocity decreased by $33.49 \%$ in the dual task and $41.69 \%$ in the triple task compared to the single-task, the degree of deviation increased by $119.18 \%$ in the dual task and $122.67 \%$ in the triple task in comparison to the single-task.

CONCLUSIONS: It was determined that changes in gait, appear when walk while using smartphones in comparison to walking without using smartphones.
\end{abstract}

Keywords: Dual-task, triple-task, spatiotemporal parameters, gait analysis

\section{Introduction}

People often perform two or more tasks simultaneously, such as speaking during walking, and dancing during singing, without being consciously aware of it [1]. The important thing in performing two or more tasks simultaneously is to establish a systematic strategy for attention distribution for each task. In the case where the attention is focused on cognitive functions, there is difficulty in motor skills, such as walking; alternatively, activation of cognitive function decreases in cases where motor skills form

*Address for correspondence: GyuChang Lee, PT, PhD, Department of Physical Therapy, Kyungnam University, 7 Kyungnamdaehak-ro, Masanhappo-gu, Changwon-si, Gyeongsangnam-do 631-701, Republic of Korea. Tel.: +82 55249 2739; Fax: +82 505999 2173; E-mail: leegc76@kyungnam.ac.kr. the focus [2]. This is called "dual-task interference." If a person's attention is concentrated on one task due to the lack of a well-established strategy, unexpected situations such as an accident may occur [2]. In particular, the attention assigned to each task is not distributed evenly when dual-tasks are performed by the elderly, which may further compromise their gait control [3]. In line with this, if too much attention is given to one task, relatively less concentration is afforded to other tasks when performing a cognitive task and a motor task at the same time [2, 4].

In general, gait is considered an automatic movement [3]. However, ambulating in crowded streets becomes a dual-task that requires cognitive function, such as attention to safety $[5,6]$. In instances where gait becomes part of a dual-task, relatively less attention is paid to gait compared to when it is performed 
as a single-task, which leads to reduced gait velocity and loss of directionality [7]. In particular, the elderly have a decreased gait velocity and cadence during a dual-task state [8], although young adults show a decrease in step length during walking on a treadmill while taking the Stroop test [9]. Most people develop an ability to perform other tasks subconsciously while walking. Nowadays, people use their mobile phones frequently, resulting in an unintentional dual-task performance. This situation sometimes may bring about the risk of unexpected accidents. Recently, several studies have been carried out to investigate changes that appear when various tasks such as walking are performed simultaneously with using mobile phones [7, 10-13]. Lamberg et al. [7] found that activation of the memory system decreases in cases of people speaking over a normal mobile phone while walking, and gait direction is biased laterally when people use text messages, which increases the distance covered. They also found that gait velocity decreases [7, 14], limited the number of words used [15], and awareness of the environment tends to decrease [11, 14-16], when people speak on the phone or send text messages while walking. In addition, it was reported that range of vision is drastically reduced for cognitive tasks undertaken during a cell phone conversation [17]. In case there is a big difference in the level of attention and cognitive steps, changes in gait appear $[2,7]$, and these actions could be very risky.

Currently, the number of smartphone users is increasing worldwide [18, 19]. A smartphone is defined as a cellular telephone with built-in applications and Internet access. It also has functions such as modern text messaging, E-mail, web browsing, video camera, MP3 player, and calling." Among these smartphone functions, the built-in applications and Internet access are the most important features for a mobile phone to be called a smartphone $[18,20]$. People can either speak on the phone or send text messages using ordinary mobile phones. However, the use of smartphones allows them to perform two or more tasks at once by making it possible to use the internet alongside the above-mentioned functions, and smartphones, with their variety of functions, lead users to perform dual-tasks in many situations. In particular, the use of smartphones while walking can lead to gait deviations by reducing cognitive abilities. Gait deviations while performing dual-tasks may increase the risk of an unpredictable accident during walking. Thus, caution should be taken when using a smartphone during walking. In previous studies, using a cell phone during walking, changes gait patterns such as deviated gait line [7]. However, in order to provide evidence supporting the hypothesis, studies investigating the changes in gait pattern while multitasking on a smartphone during walking have not yet been conducted.

Therefore, the current study was designed to investigate changes in gait pattern that occur because of the complexity of using smartphones during walking.

\section{Methods}

\subsection{Participants}

Twenty-six healthy adults aged $18-30$ years who attended the university participated in the current study. An advertisement recruiting participants was made using a school notice board, and 30 volunteers were recruited. The volunteers were screened by a researcher on the basis of the following inclusion: (1) those who usually use smartphones; (2) those who have no problem with visual or auditory functions; and (3) those without musculoskeletal injuries or diseases affecting gait. People with cognitive impairment, alcohol or drug abuse records, or those taking medications that could affect gait were excluded as subjects for the study. Two participants with musculoskeletal injuries did not meet the criteria, and they were excluded as subjects for the study. Additionally, two participants did not attend the measurement for gait pattern. Therefore, 26 subjects participated in the study. All participants signed informed consent forms after receiving a detailed explanation of the study. The procedures were approved by the Kyungnam University Institutional Review Board. The general characteristics of the participants were as follows: 26 participants consisting of 16 men and 10 women with a mean age of 21.73 years, mean height of $169.50 \mathrm{~cm}$, and mean weight of $62.12 \mathrm{~kg}$. The characteristics for experience using a smartphone of participants were described in the Table 1.

\subsection{Procedure}

The investigation for changes in gait pattern according to task was conducted a week after the recruitment of participants. Tasks performed in this study were as follows: The first was a single-task routine of walking toward a fixed target, and the second was a dual-task routine of finding and downloading an Application (App) using a smartphone while walking toward a fixed target. The third was a triple-task routine of downloading an App and speaking over the 
Table 1

Smartphone use experience of participants

\begin{tabular}{lcc}
\hline \multicolumn{1}{c}{ Characteristics } & Number $(\%)$ \\
\hline Duration of smartphone use & $<3$ months & $0(0 \%)$ \\
& $<6$ months & $0(0 \%)$ \\
& $<1$ year & $3(11.5 \%)$ \\
& $<2$ year & $11(42.3 \%)$ \\
& $>2$ year & $12(46.2 \%)$ \\
Smartphone use per day & $<30$ minute & $1(3.8 \%)$ \\
& $<1$ hour & $2(7.7 \%)$ \\
& $<2$ hours & $8(30.8 \%)$ \\
& $<4$ hours & $10(38.5 \%)$ \\
Type of smartphone & $>4$ hours & $5(19.2 \%)$ \\
& IOS based & $12(46.2 \%)$ \\
Main purpose of smartphone use & android based & $14(53.8 \%)$ \\
& web browsing & $8(30.8 \%)$ \\
& calling & $4(15.4 \%)$ \\
& SMS & $14(53.8 \%)$ \\
\hline
\end{tabular}

smartphone while walking toward a fixed target. Each task was performed three times, and a minute rest was allowed between each task. The order of tasks to be performed was assigned randomly by a researcher using a number table. Participants were required to perform each task while walking a 10-meter distance. Participants were shown a mark placed on the wall at eye level about 10-meter away before walking, and were instructed to walk toward the mark. Participants were then instructed to focus on a secondary task rather than gait and walk comfortably. While walking, they were instructed to keep performing the secondary task of finding and downloading an App before they reached the target. A set of 10 applications that were simple but required concentration and had titles of more than six syllables were identified to be randomly selected by the researchers (Appendix). In the triple-task, while finding and downloading the App as in the dual-task, participants were also asked to answer questions put by researchers using the phone feature of the smartphones. Thirteen questions requiring sufficient cognitive effort, while also being easily answered by most people, were determined in advance and then randomized and assigned (Appendix). In addition, participants were allowed to use earphones when performing the triple-task for clearer telephone conversation, and a researcher who asked the questions via telephone were located in a different place so that they would not interfere with the participants' gait. The participants were allowed to get plenty of rest when complaints over inconveniences were raised during the experiment. All the experiments were conducted in a quiet and spacious laboratory for physical therapy. For tasks such as finding applications and holding a telephone conversation, smartphones (Galaxy S, Samsung, Seoul,
Korea) were used. All participants were taught how to find applications and talk with the smartphones, and allowed a single trial prior to the formal performance of the tasks.

\subsection{Measurements}

The spatiotemporal parameters of gait were measured by means of GAITRite (GAITRite Electronic Walkway. CIR Systems Inc., NY, USA). Variables such as gait velocity, cadence, step length, stride length, and double support time were also measured. The GAITRite has a length of $4.8 \mathrm{~m}$ and width of $0.9 \mathrm{~m}$. It is an electronic walkway that contains sensors arranged in a pattern to measure the pressure exerted by the feet on the contact surface. The equipment acquires data, which are transmitted via connections to a laptop; application software within the computer processes patterns from the soles of the feet. Participants walked about 10-meter, which included the length of the GAITRite. The GAITRite was installed about 1-meter past the starting line to reduce participants' awareness of the equipment. At a researcher signal, participants were to ambulate a distance of 10-meter while performing the assigned task. For each task, 3 attempts were made. In cases where data acquisition was not accurate, re-performance was requested so that 3 sets of data could be collected. The test-retest reliability of the GAITRite was $0.902-0.970$, and the inter-rater reliability was $0.945-1.000$ [21].

To determine the degree of lateral deviation of gait, after connecting the starting and ending points of the 10-meter distance with a straight line, the deviation distance was measured against the intended path by a straight line extending from the starting point and marking the ending point of the participants' actual gait with a dot [7]. The distance from the starting point to the ending point for gait was measured by marking the point at which participants stopped at the end of 10-meter, where the bottom of the middle of the heel intersected the floor. The degree of lateral deviation was determined as in an experiment to measure changes in gait by means of GAITRite. In any case of failure to acquire accurate data, re-measurement was conducted without giving separate feedback to participants. The degree of lateral deviation was measured as following [7] (Fig. 1).

\subsection{Data analysis}

For data analysis, SPSS 12.0 (IBM Corporation, Armonk, NY) was used. Data on the degree of 


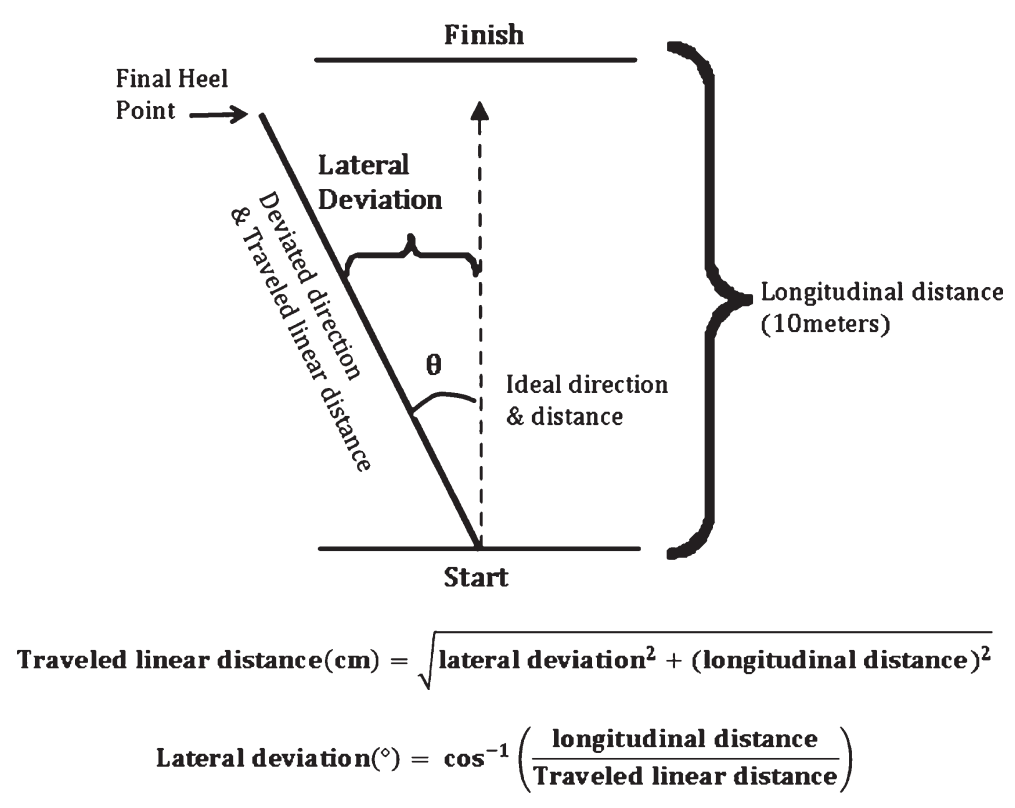

Fig. 1. Illustration for measurement of lateral deviation.

deviation and spatiotemporal parameters of the gait were collected. All the data except for double support time and deviation were normally distributed. The normally distributed data were analyzed using one-way ANOVA to compare the differences between the 3 conditions, and Tukey's post-hoc test was conducted. Since double support time and deviation were not normally distributed, they were analyzed using Kruskal-Wallis, a non-parametric analysis, and the Mann-Whitney U test was used. The level of statistical significance was set at 0.05 .

\section{Results}

Significant differences were found between the single-task and dual-task, and the single-task and triple-task in velocity, cadence, step length, stride length, double support time, and deviation $(p<0.05)$ (Table 2). However, a significant difference was not found between the dual-task and triple-task in all variables (Table 2). In particular, gait velocity decreased by $33.49 \%$ in the dual-task and $41.69 \%$ in the triple-task compared with the single-task, cadence decreased by $17.65 \%$ in the dual-task and $26.89 \%$ in the triple-task compared with the single-task, and the degree of deviation increased by $119.18 \%$ in the dualtask and $122.67 \%$ in the triple-task compared with the single-task (Table 2).

\section{Discussion}

This study was to investigate changes in gait pattern during multitasks with using a smartphone. The results showed that there was a significant change in gait in a situation where two or more tasks using a smartphone are given in comparison to a singletask of only performing gait. However, there was no significant difference when the number of tasks was increased from two to three.

Lamberg et al. [7] found that gait velocity decreases by $16 \%$ when people walk using smartphones compared to only walking, and gait velocity decreases by $33 \%$ when they walk while sending text messages [7]. This study also found that gait velocity decreases by $33.49 \%$ if ambulating while finding and installing applications using a smartphone compared to that of ambulation only. In addition, a reduction in gait velocity of $41.69 \%$ appeared in a triple-task using a smartphone in more complex ways in comparison to the single-task. These results show that dual-task performance brings about a reduction in gait velocity compared to a single-task performance. In cases where several tasks were performed at the same time, a systematic and efficient strategy for distribution of attention is required. On the basis of this strategy, the participants' reduction in gait velocity appears to occur in order to maintain stability, resulting in a decrease of gait efficiency. Although there was no 
Table 2

Changes of gait parameters

\begin{tabular}{|c|c|c|c|c|c|c|}
\hline Variables & Single-task & Dual-task & Triple-task & $\begin{array}{l}\text { Difference between } \\
\text { single and dual }\end{array}$ & $\begin{array}{l}\text { Difference between } \\
\text { single and triple }\end{array}$ & $p$ \\
\hline Velocity $(\mathrm{m} / \mathrm{sec})$ & $111.72 \pm 22.60$ & $74.31 \pm 20.53^{*}$ & $65.14 \pm 26.03^{\dagger}$ & $-33.49 \%$ & $-41.69 \%$ & 0.000 \\
\hline Cadence (numbers of step/min) & $110.32 \pm 11.62$ & $90.85 \pm 15.54^{*}$ & $80.65 \pm 18.11^{\dagger}$ & $-17.65 \%$ & $-26.89 \%$ & 0.000 \\
\hline Step length $(\mathrm{cm})$ & $60.12 \pm 7.78$ & $48.13 \pm 7.13^{*}$ & $45.33 \pm 9.36^{\dagger}$ & $-19.95 \%$ & $-24.60 \%$ & 0.000 \\
\hline Stride length $(\mathrm{cm})$ & $120.91 \pm 16.12$ & $92.93 \pm 14.11^{*}$ & $91.50 \pm 18.16^{\dagger}$ & $-23.14 \%$ & $-24.32 \%$ & 0.000 \\
\hline Double support time (sec) & $0.27 \pm 0.05$ & $0.49 \pm 0.20^{*}$ & $0.57 \pm 0.31^{\dagger}$ & $81.48 \%$ & $111.11 \%$ & 0.000 \\
\hline Deviation $(\mathrm{cm})$ & $1.72 \pm 0.00$ & $3.77 \pm 2.38^{*}$ & $3.83 \pm 3.58^{\dagger}$ & $119.18 \%$ & $122.67 \%$ & 0.004 \\
\hline
\end{tabular}

* Significant differences between single-task and dual-task. ${ }^{\dagger}$ Significant differences between single-task and triple-task.

significant difference, gait velocity did decrease by $20 \%$ in a triple-task with more multiple and complex performances in comparison with a dual-task. In the triple-task, participants were instructed to walk while having a telephone conversation, in addition to finding and installing applications using smartphones. A telephone conversation requires the ability to produce language, along with vocalization. This requirement interferes with gait, which leads to a further decrease in gait velocity [22-24]. That's because the more complex the task, the greater the difficulty in performing it [2], and entering characters for finding applications is complicated [7] and further requires the cognitive ability to produce language. As a result, the attention to gait further decreased, and a difference in gait velocity occurred. It is expected that if only vocalization, a cognitive activity, is processed preferentially, concentration on gait stability decreases, and the risk of falls may increase [3].

Even in other spatiotemporal parameters such as cadence, step length, stride length and double support time, significant differences appeared between the single-task and dual-task, as well as between the single-task and triple-task. As the tasks multiply and become more complex, cadence, step length and stride length decreases, and double support time increases, which may be attributed to the fact that attention is assigned to other tasks, and so attention to gait decreases. That is, changes in gait pattern occurred to create stable walking by compensating for the reduced attention [25]. This gait pattern is similar to that of many elderly people, since it is characterized by fewer strides, shortened step length and stride length, and a widened base of support in comparison to young adults [25]. The elderly were also found to adopt a gait pattern that would stabilize walking to compensate for their decreased ability to balance [25]. In this regard, it can safely be said that the changes in gait pattern that appear in this study endeavor to stabilize gait by offsetting the attention distributed to other tasks.
As for the degree of gait deviation, there was a difference of $119.18 \%$ between the single-task and dual-task, and a difference of $122.67 \%$ between the dual-task and triple-task. In the study designed to perform the task of using a general mobile phone while walking, Lamberg et al. [7] found that about $61 \%$ of all participants deviated laterally when they walked while talking over the telephone, and their movement increased by $13 \%$. In cases where people are walking while using a smartphone, more concentration is on the smartphone than on the gait pattern [7]. Therefore, they proceed with gait by relying on the memory of the target seen temporarily, instead of keeping an eye on the target. In such cases, visual attention is focused on the smartphone. The direction of the target captured by transient sight is felt to be farther than its actual location, which is called attentional repulsion effect [26]. In addition, maintaining the observed target in the head weakens when vision is limited [26]. Due to this limitation, participants in this study experienced visual distortion and poor estimation of space, thereby contributing to the deviation in the direction of gait [26], which in turn made it difficult for them to be aware of surrounding circumstances, resulting in losing directionality. Losing directionality means the length of forward progression becomes longer than originally anticipated.

The results of this study show that using a smartphone while walking leads to changes in gait patterns. However, the study has several limitations. First, since participants did not use their own smartphones, the degree of difficulty in performing the task might have been different among participants. In addition, participants who use the same smartphone as the one used in the study could have performed the task better than those who did not. Second, because the sample size was small, the results should not be generalized to all situations and caution must be taken in interpreting the results. Lastly, the cognitive changes assigned to each task were not investigated in this study. If the cognitive changes are also investigated, the degree 
of attention distribution that appears when a motor task and a cognitive task are performed simultaneously will be identified. On the basis of these results, it is possible to present the degree of distribution of multitask performed simultaneously during walking.

\section{Conflict of interest}

The authors have no conflict of interest to declare.

\section{References}

[1] Yang YR, Chen YC, Lee CS, Cheng SJ, Wang RY. DualTask-related gait changes in individuals with stroke. Gait and Posture 2007;25(2):185-90.

[2] Armieri A, Holmes JD, Spaulding SJ, Jenkins ME, Johnson AM. Dual-task performance in a healthy young adult population: Results from a symmetric manipulation of task complexity and articulation. Gait and Posture 2009;29(2): 346-8.

[3] Siu KC, Chou LS, Mayr U, van Donkelaar P, Woollacott MH. Attentional mechanisms contributing to balance constraints during gait: The effects of balance impairments. Brain Research 2009;1248:59-67.

[4] Kelly VE, Janke AA, Shumway-Cook A. Effects of instructed focus and task difficulty on concurrent walking and cognitive task performance in healthy young adults. Experimental Brain Research 2010;207(1):65-73.

[5] Yogev-Seligmann G, Hausdorff JM, Giladi N. The role of executive function and attention in gait. Movement Disorders 2008;23(3):329-42.

[6] Shumway-Cook A, Woollacott MH. Motor control: Translating research into clinical practice, 4 th ed. Baltimore: Lippincott Williams and Wilkins, 2012.

[7] Lamberg EM, Muratori LM, Muratori. Cell phones change the way we walk. Gait and Posture 2012;35(4):688-90.

[8] Toulotte C, Thevenon A, Watelain E, Fabre C. Identification of healthy elderly fallers and non-fallers by gait analysis under dual-task conditions. Clinical Rehabilitation 2006;20:269-76.

[9] Grabiner MD, Troy KL. Attention demanding tasks during treadmill walking reduce step width variability in young adults. Journal of Neuroengineering and Rehabilitation 2005;2:25

[10] Stavrinos D, Byington KW, Schwebel DC. Distracted walking: Cell phones increase injury risk for college pedestrians. Journal of Safety Research 2011;42(2):101-7.

[11] Neider MB, McCarley JS, Crowell JA, Kaczmarski H, Kramer AF. Pedestrians, vehicles, and cell phones. Accident; Analysis Prevention 2010;42(2):589-94.
[12] Stavrinos D, Byington KW, Schwebel DC. Effect of cell phone distraction on pediatric pedestrian injury risk. Pediatrics 2009;123(2):179-85.

[13] Schwebel DC, Stavrinos D, Byington KW, Davis T, O’Neal EE, De-Jong D. Distraction and pedestrian safety: How talking on the phone, texting, and listening to music impact crossing the street. Accident; Analysis Prevention 2012;45:266-7.

[14] Hatfield J, Murphy S. The effects of mobile phone use on pedestrian crossing behavior at signalized and unsignalized intersections. Accident; Analysis Prevention 2007;39(1):197-205

[15] Nasar J, Hecht P, Wener R. Mobile telephones, distracted attention, and pedestrian safety. Accident; Analysis Prevention 2008;40(1):690-1.

[16] Bungum TJ, Day C, Henry J. The association of distraction and caution displayed by pedestrians at a lighted crosswalk. Journal of Community Health 2005;30(4):269-79.

[17] Maples WC, DeRosier W, Hoenes R, Bendure R, Moore S. The effects of cell phone use on peripheral vision. Optometry 2008;79(1):36-42.

[18] Hsiao MH, Chen LC. Smart Phone Demand: An Empirical Study on the Relationships between Phone Handset, Internet Access and Mobile Services. Telematics and Informatics 2015;32(1):158-68.

[19] Alberda W, Kampinga O, Kassels R, van Kester R, Noriega J, Vink P. Access improvement to aircraft passengers' hand luggage. Work: A Journal of Prevention, Assessment and Rehabilitation 2015;50(4):659-67.

[20] Prata W, de Moraes A, Quaresma M. User's demography and expectation regarding search, purchase and evaluation in mobile application store. Work: A Journal of Prevention, Assessment and Rehabilitation 2012;41:1124-31.

[21] McDonough AL, Batavia M, Chen FC, Kwon S, Ziai J. The validity and reliability of the GAITRite system's measurements: A preliminary evaluation. Archives of Physical Medicine and Rehabilitation 2001;82(3):419-25.

[22] Dault MC, Yardley L, Frank JS. Does articulation contribute to modifications of postural control during dual-task paradigms? Brain Research. Cognitive Brain Research 2003;16(3):434-40.

[23] Yardley L, Gardner M, Leadbetter A, Lavie N. Effect of articulatory and mental tasks on postural control. Neuroreport 1999;10(2):215-9.

[24] Plummer-D'Amato P, Altmann LJP, Saracino D, Fox E, Behrman AL, Marsiske M. Interactions between cognitive tasks and gait after stroke: A dual-task study. Gait and Posture 2008;27(4):683-8.

[25] Siu KC, Chou LS, Mayr U, van Donkelaar P, Woollacott $\mathrm{MH}$. Attentional mechanisms contributing to balance constraints during gait: The effects of balance impairments. Brain Research 2009;1248:59-67.

[26] Au RK, Ono F, Watanabe K. Spatial distortion induced by imperceptible visual stimuli. Conscious and Cognition 2012;22(1):99. 


\section{Appendix}

Applications and questions used for participants

Smartphone applications used by participants while walking

Naver-Wingspoon

Monster Life

Smart Bill

Mobile T-money

Smurf Village Guide

Google Maps- Street View

Horizontal and Vertical Word Quiz

ASTRO File Manager

Real Changwon Bus

Phone Decorating Application Heaven

Questions given by phone to participants while walking

Memorize multiplication facts of 6

Memorize multiplication facts of 7

Memorize multiplication facts of 8

Memorize multiplication facts of 9

Subtract by 7 , starting with 100

State the names of all provinces and capital cities

State the name some countries in the world

State the name some animals you know

State the name some fruits you know

State the name some plants you know 